\title{
Effect of Caesarean Section on Neonatal Health in Bangladesh after Controlling Selection Bias: Propensity Score Based Analysis
}

\author{
Md. Mahmudur Rahman, Sabina Sharmin* and Taslim Sazzad Mallick \\ Department of Statistics, University of Dhaka, Dhaka-1000, Bangladesh
}

(Received: 5 May 2020; Accepted: 24 February 2021)

\begin{abstract}
The paper examines the effect of caesarean section (C-section) on early neonatal mortality, neonatal mortality, and early initiation of breastfeeding using Bangladesh Demographic and Health Survey (BDHS), 2014 data. Propensity score matching and weighting methods were used to estimate unbiased estimate of treatment effect. The study demonstrates how conclusion about treatment effect varies with and without having balance in the treatment groups. Standard analysis, without caring about balance, reveals that Csection has no significant impact on early neonatal mortality and neonatal mortality. After applying propensity score adjusted methods, balance was achieved in the treatment groups and it was found that C-section has significant effect on early neonatal mortality and neonatal mortality. However, there was no difference between standard and PS adjusted methods in estimating the effect of C-section on early initiation of breastfeeding. It is concluded that children who were delivered by $\mathrm{C}$-section have significantly lower odds of early neonatal mortality, neonatal mortality, and early initiation of breastfeeding as compared to the children who were not delivered by C-section.
\end{abstract}

Keywords: Propensity score, Neonatal health, Caesarean section

\section{Introduction}

In Bangladesh, the percentage of caesarean -section (Csection) delivery has rapidly increased in recent years. Bangladesh Demographic and Health Survey (BDHS) ${ }^{1}$, 2014 shows that $23 \%$ of live births in the three years preceding the survey were delivered by $\mathrm{C}$-section. It also reflects that 6 in every 10 births in a health facility are delivered by $\mathrm{C}$-section.

Very low rate of C-section delivery may reflect women's lack of access to skilled care for complicated deliveries, whereas, very high rate of $\mathrm{C}$-section delivery may indicate overuse of the c-section delivery, along with unnecessary surgical risk ${ }^{2}$.

Researchers found that women who deliver by c-section delivery are less likely to breastfeed or delay breastfeeding initiation (Rowe-Murray et. al, 2002) ${ }^{3}$.

Macdorman et al. ${ }^{4}$ (2006) showed that neonatal mortality is increased more than two-fold after birth by cesarean, even after excluding infants with congenital anomalies and presumed intrapartum hypoxic events (Apgar score $<4)$ and adjusting for demographic and medical covariates.

The percentage of c-section delivery is considered as an indicator of access to life-saving services for both mothers and newborns. There is a growing interest in the study of measuring the effect of $\mathrm{C}$-section on different health related outcomes.

\section{Objective of the Study}

This paper examines the effect of caesarean section (Csection) on early neonatal mortality, neonatal mortality, and early initiation of breastfeeding using Bangladesh Demographic and Health Survey (BDHS), 2014 data.

\footnotetext{
*Author for correspondence. e-mail: sabina.sharmin@du.ac.bd

\section{Design of the study}

Historically, researchers depended on the use of regression adjustment to control the effect of measured and unmeasured pretreatment variables.

In observational study, researchers draw causal inference based on what they observe. Researchers do not manipulate the assignment of individuals into either a treatment group or a control group in this set up. It is quite possible that the characteristics of pretreatment variables of treatment group will differ from the control group. Rosenbaum \& Rubin ${ }^{5}$ (1983) showed that the probability that an individual would belong to either treatment group or control group is determined as a function of the measured covariates for that individual. Therefore, the conclusion drawn from observational study may be misleading since there is a possibility that the real effect might come from measured or unmeasured confounder(s) i.e., variable(s) that effect both outcome and treatment. Propensity score analysis overcomes the limitations of observational study, by enabling us to make causal inference from observational data.

Propensity score is the probability of receiving a treatment conditional on a set of observed covariates (Rosenbaum \& Rubin, 1983), which is defined as $e(X)=p(Z=1 \mid X)$, where $Z$ is the treatment and $X$ is the observed pretreatment variables.

For binary or dichotomous treatment variable, let us consider $\mathrm{Z}$ be a treatment indicator, if $Y_{i}(1) \& Y_{i}(0)$ represent the potential outcome of the individual that assigned to the treatment group and the individual that assigned to the control group, respectively. Then, the observed outcome is defined as, $Y_{i}=Z_{i} Y_{i}(1)+$ $\left(1-Z_{i}\right) Y_{i}(0)$.

Propensity score methods are used to create a randomized environment of Randomized Control Trial (RCT) so that a meaningful causal inference can be drawn. By conditioning 
the propensity score, balance may be achieved for the pretreatment variables in the treatment group and control group. Thus, for the set of individuals who have same propensity scores; have same distribution of observed pretreatment variables between the treated individuals and untreated individuals.

The main difference between RCT and observational study is the randomization property. Because of the randomization process, the influence of confounding factors is cancelled out in RCT. There remains no systematic difference in measured and unmeasured pretreatment variables between treatment and control groups.

Balancing pretreatment variables prior to the estimation of treatment effect on outcome is the most important concern in propensity score analysis. If the treatment groups are properly balanced, then it can be said that each of the groups have equal chance of receiving treatment, thus the randomized property of RCT is justified. In addition, an unbiased treatment effect can be obtained.

In randomized controlled trial (RCT), means or proportions of individuals who experienced the outcome between treatment group and control group may be directly compared. This is because, individuals in two groups are chosen in a way that they have similar background. Besides, individuals are assigned to the treatment groups at random, and so both measured and unmeasured covariates are balanced among the treatment groups.

In RCT, true propensity score is known whereas in observational study, it is unknown, therefore it is estimated from the data. The most commonly used tools for estimating propensity scores are logistic regression and generalized boosted models in which treatment variables is regressed on observed pretreatment variables. There are several techniques used to estimate the propensity score, such as the use of bagging or boosting (Lee et al. ${ }^{6}, 2010$; McCaffrey et al. $\left.{ }^{7}, 2004\right)$, recursive partitioning or treebased methods (Lee et al., 2010; Setoguchi et al. ${ }^{8}, 2008$ ), random forests (Lee et al., 2010), and neural networks (Setoguchi et al., 2008).

Four different propensity score methods can be used to remove the effects of confounding and achieve balance among the treatment groups when estimating the effects of treatment on outcomes. The methods are as follows:

1. propensity score matching

2. stratification on the propensity score

3. propensity score weighting

4. covariate adjustment using the propensity score

Over the past decade, Hong $\& \mathrm{Yu}^{9}$ (2008) used PS methods to assess the effects of kindergarten retention on children's social-emotional development. Using these methods, Ye \& Kaskutas $^{10}$ (2009) examined the effectiveness of Alcoholics Anonymous, while Wyse et al. ${ }^{11}$ (2008) measured the effects of small school size on mathematics achievement. To assess the effect of teenage alcohol use on education attainment (Staff et al. ${ }^{12}, 2008$ ), the impact of candidemia on excess mortality, increased length of stay, and the burden of cost of hospitalization (Zaoutis et al. ${ }^{13}, 2005$ ), the relationship between adolescent marijuana use and adult outcomes (Stuart et al. ${ }^{14,} 2008$ ), propensity score methods are used.

In this study, to examine the effect of caesarean section $(\mathrm{C}$ section) on early neonatal mortality, neonatal mortality, and early initiation of breastfeeding - propensity score matching and weighting methods were used to estimate the true treatment effect.

\section{Data and Variables}

Bangladesh Demographic and Health Survey (BDHS), 2014 data was used which has one record for every child of eligible women who born in the last five years. Mother of each of these children is included in the data. Child health indicators such as immunization coverage, vitamin A supplementation, and recent occurrences of diarrhea, fever, and cough for young children and treatment of childhood diseases as well as fertility and mortality rates are focused in the dataset. Children of women born in the last 5 years (0-59 months) are the unit of analysis (case) in this dataset.

The following variables were considered for this study: early neonatal mortality, neonatal mortality, early initiation of breastfeeding, delivery by c-section, type of place of residence, region or division, mother's education level, mother's age at birth, wealth index, birth order number, sex of child, religion, exposure to media, NGO membership of mother and awareness of community health clinic. Only the children who were born in the preceding five years of the survey were considered and 7886 observations were found from Children Recode dataset.

To examine the effect of c-section on mortality a sample of 4733 observations were used from 7886 observations after removing the missing values in the variable "Delivery by csection".

Another sample of 4335 observations from 7886 observations after removing the missing values in the variable "Early initiation of breastfeeding" was prepared to estimate the effect of c-section on early initiation of breastfeeding in Bangladesh.

\section{Propensity Score Methods: Weighting Versus Matching}

\section{Weighting}

Three types of weights were used in this study. A brief description is given below.

Stabilized weight (w.boost): w.boost is defined as

$$
w . \text { boost }=\left\{\begin{array}{cl}
1, & \text { if } Z=1 \\
\frac{e(X)}{1-e(X)}, & \text { if } Z=0
\end{array}\right.
$$

Here, $e(X)$ is the estimated propensity scores and $Z$ is the treatment indicator. Here, weight $=1$ is given to the 
individuals who belong to the treatment group and weight $=$ $\frac{e(X)}{1-e(X)}$ to the individuals who belong to the control group.

Standardized Mortality Ratio Weight (w.smrw): This is defined as one for the individuals who are treated and the ratio of the estimated propensity score to one minus the estimated propensity score for those who are not treated. w.smrw is same as the w.boost. The difference is that the propensity score is estimated through logistic regression model.

Inversed Probability of Treatment Weight (w.iptw): IPTW is defined as the inverse of the estimated propensity score for the individuals in the treatment group and the inverse of one minus the estimated propensity score for the individuals in the control group. w.iptw can be expressed as

$$
\text { w. iptw }=\left\{\begin{array}{cl}
\frac{1}{e(X)}, & \text { if } Z=1 \\
\frac{1}{1-e(X)}, & \text { if } Z=0
\end{array}\right.
$$

\section{Matching}

For the purpose of the study, nearest neighbor matching within a specified caliper distance were used. A caliper of width equal to 0.2 of the standard deviation of the logit of the propensity score were used to minimize the mean squared error of the estimated treatment effect in several scenarios.

\section{Results}

The propensity scores are estimated through generalized boosted model. R package 'Twang' was used to get these scores. After estimating the propensity scores, the first step is to check whether balance is achieved between treatment and pretreatment variables in the PS adjusted data. If the balance is achieved, there will not be any significant difference in receiving treatment among different categories of pretreatment variables. Table 1 and 2 show the proportion difference in the treatment groups for all pretreatment variables for assessing the balance between the treatment group and control group for each of the pretreatment variables in both mortality-based sample and breastfeeding-based sample, respectively.

Table 1. Balance Assessment: Proportion difference before and after adjustment by PS (mortality-based sample)

\begin{tabular}{|c|c|c|c|c|c|c|}
\hline \multirow[t]{2}{*}{ Variables } & \multirow[t]{2}{*}{ Categories } & \multirow[t]{2}{*}{ Unadjusted } & \multicolumn{4}{|c|}{ Adjusted proportion difference } \\
\hline & & & w.boost & w.smrw & w.iptw & matching \\
\hline \multirow[t]{2}{*}{ Place of residence } & Urban & $.2343 *$ & .0023 & .0086 & -.0018 & .0518 \\
\hline & Rural & - & - & - & - & - \\
\hline \multirow[t]{7}{*}{ Region } & Barisal & -.0247 & -.0004 & .0016 & -.0100 & .0030 \\
\hline & Chittagong & -.0410 & -.0041 & -.0062 & .0074 & .0040 \\
\hline & Dhaka & .0873 & .0039 & .0057 & -.0072 & .0040 \\
\hline & Khulna & .0729 & .0088 & -.0046 & .0177 & -.0169 \\
\hline & Rajshahi & .0237 & -.0067 & .0005 & -.0055 & -.0070 \\
\hline & Rangpur & -.0249 & -.0007 & -.0005 & .0152 & .0020 \\
\hline & Sylhet & .0933 & -.0008 & .0034 & -.0177 & .0110 \\
\hline \multirow[t]{4}{*}{ Mother's education level } & No education & $-.1238 *$ & -.0001 & -.0003 & -.0202 & -.0060 \\
\hline & Primary & $-.1787 *$ & -.0003 & -.0055 & .0253 & -.0080 \\
\hline & Secondary & .0872 & -.0089 & -.0070 & -.0059 & -.0249 \\
\hline & Higher & $.2153 *$ & .0092 & .0129 & .0008 & .0269 \\
\hline \multirow[t]{3}{*}{ Mother's age at birth } & $<20$ & -.0403 & -.0026 & -.0094 & .0025 & -.0090 \\
\hline & $20-35$ & .0478 & .0024 & .0113 & .0091 & .0030 \\
\hline & $>35$ & -.0074 & .0002 & -.0018 & -.0115 & .0060 \\
\hline \multirow[t]{3}{*}{ Wealth index } & Poor & $-.3380 *$ & -.0032 & -.0024 & -.0090 & -.0189 \\
\hline & Middle & -.0448 & -.0009 & -.0049 & .0100 & -.0090 \\
\hline & Rich & $.3829 *$ & .0041 & .0073 & -.0009 & .0279 \\
\hline \multirow[t]{2}{*}{ Birth order number } & $1-2$ & $.1642 *$ & -.0010 & -.0023 & .0258 & .0120 \\
\hline & $3+$ & - & - & - & - & - \\
\hline \multirow{2}{*}{ Sex of child } & Male & .0329 & -.0080 & -.0103 & .0091 & .0120 \\
\hline & Female & - & - & - & - & - \\
\hline \multirow[t]{2}{*}{ Religion } & Islam & -.0210 & -.0035 & -.0004 & .0027 & -.0139 \\
\hline & Other religion & - & - & - & - & - \\
\hline \multirow{2}{*}{ Exposure to media } & Unexposed & - & - & - & - & - \\
\hline & Exposed & $.2775 *$ & -.0001 & .0008 & .0129 & .0090 \\
\hline \multirow{2}{*}{ NGO membership of mother } & No & - & - & - & - & - \\
\hline & Yes & -.0395 & .0048 & -.0019 & .0244 & -.0060 \\
\hline \multirow[t]{2}{*}{ Aware of community clinic } & No & - & - & - & - & - \\
\hline & Yes & -.0627 & .0018 & .0058 & .0001 & -.0080 \\
\hline
\end{tabular}

* Not Balanced $(p$-value $<0.05)$ 
Table 2. Balance Assessment: Proportion difference before and after adjustment by PS (Breastfeeding-based sample)

\begin{tabular}{|c|c|c|c|c|c|c|}
\hline \multirow[t]{2}{*}{ Variables } & \multirow[t]{2}{*}{ Categories } & \multirow[t]{2}{*}{ Unadjusted } & \multicolumn{4}{|c|}{ Adjusted proportion difference } \\
\hline & & & w.boost & w.smrw & w.iptw & matching \\
\hline \multirow{2}{*}{ Place of residence } & Urban & $.2361 *$ & .0041 & .0124 & -.0039 & .0273 \\
\hline & Rural & - & - & - & - & - \\
\hline \multirow[t]{7}{*}{$\overline{\text { Region }}$} & Barisal & -.0261 & -.0011 & .0014 & -.0094 & -.0011 \\
\hline & Chittagong & -.0435 & -.0017 & -.0049 & .0046 & .0021 \\
\hline & Dhaka & .0894 & .0023 & .0030 & -.0049 & .0032 \\
\hline & Khulna & .0685 & .0056 & -.0050 & .0173 & .0011 \\
\hline & Rajshahi & .0250 & -.0043 & .0024 & -.0074 & -.0221 \\
\hline & Rangpur & -.0285 & .0003 & -.0002 & .0168 & .0063 \\
\hline & Sylhet & -.0846 & -.0011 & .0034 & -.0170 & .0105 \\
\hline \multirow[t]{4}{*}{ Mother's education level } & No education & $-.1214 *$ & -.0001 & -.0004 & -.0169 & -.0042 \\
\hline & Primary & $-.1824 *$ & -.0003 & -.0060 & .0247 & -.0126 \\
\hline & Secondary & .0843 & -.0092 & -.0062 & -.0081 & -.0126 \\
\hline & Higher & $.2196 *$ & .0096 & .0126 & .0003 & .0294 \\
\hline \multirow[t]{3}{*}{ Mother's age at birth } & $<20$ & -.0414 & -.0006 & -.0090 & .0047 & -.0011 \\
\hline & $20-35$ & .0512 & .0003 & .0112 & .0062 & .0063 \\
\hline & $>35$ & -.0098 & .0003 & -.0022 & -.0109 & .0074 \\
\hline \multirow[t]{3}{*}{ Wealth index } & Poor & $-.3339 *$ & -.0020 & -.0031 & -.0058 & -.0294 \\
\hline & Middle & -.0499 & -.0013 & -.0054 & .0109 & -.0126 \\
\hline & Rich & $.3838 *$ & -.0033 & .0085 & -.0051 & .0420 \\
\hline \multirow{2}{*}{ Birth order number } & $1-2$ & $.1671^{*}$ & .0000 & -.0013 & .0213 & .0074 \\
\hline & $3+$ & - & - & - & - & - \\
\hline \multirow[t]{2}{*}{ Sex of child } & Male & .0330 & -.0079 & -.0134 & .0119 & -.0189 \\
\hline & Female & - & - & - & - & - \\
\hline \multirow{2}{*}{ Religion } & Islam & -.0193 & -.0045 & -.0016 & .0028 & .0000 \\
\hline & Other religion & - & - & - & - & - \\
\hline \multirow{2}{*}{ Exposure to media } & Unexposed & - & - & - & - & - \\
\hline & Exposed & $.2755^{*}$ & -.0013 & .0008 & .0125 & .0210 \\
\hline \multirow{2}{*}{ NGO membership of mother } & No & - & - & - & - & - \\
\hline & Yes & -.0441 & .0032 & -.0038 & .0201 & -.0168 \\
\hline \multirow[t]{2}{*}{ Aware of community clinic } & No & - & - & - & - & - \\
\hline & Yes & -.0622 & .0000 & .0036 & .0003 & .0126 \\
\hline
\end{tabular}

* Not Balanced ( $p$-value $<0.05)$

From the above tables, it was found that place of residence (Urban), mother's education level (No education, Primary, Higher), wealth index (Poor, Rich), birth order number (1-2) and exposure to media are not balanced in unadjusted method. That means out of 24 binary variables 8 binary variables are found not balanced. However, adjusting by three weights and by matching, it is found that all the binary variables are properly balanced for each of the four methods.

\section{Effect of c-section on early neonatal mortality}

Table 3 shows the Odds Ratio (OR) of c-section on early neonatal mortality using logistic regression model for unadjusted data and different propensity score adjusted data along with corresponding statistical significance.

Adjusted by w.boost and w.smrw, it was found that csection has significant effect on early neonatal mortality at $10 \%$ level of significance. The OR obtained from the above two method is .55 i.e. a child who was given birth by csection delivery has $45 \%$ lower odds of having the early neonatal death than a child who was not given birth by csection delivery, keeping all other covariates at a fixed level. w.boost and w.smrw gave similar results in this case.

Applying w.iptw, it was observed that a non-c-section child is 1.47 times more likely to have early neonatal death compared to a c-section child, keeping all other covariates at a fixed level. There is a significant association between c-section delivery and early neonatal mortality at 5\% level of significance.

In matching method, it was found that significant effect of c-section on early neonatal mortality at 5\% level of significance. The result shows that a non-c-section child is 2.17 times as likely as to have early neonatal death compared to a c-section child, keeping all other covariates at a fixed level.

Although the standard logistic regression shows an insignificant association between c-section and early neonatal mortality, after achieving proper balance in the treatment groups of the pretreatment variables using propensity adjusted methods, significant association was found.

From this result, it is concluded that sometimes the true effect cannot be achieved because of the effect of confounder. But, if the effect of confounders can be controlled by achieving proper balance for the pretreatment variables using appropriate balance technique, the true treatment effect on outcome can be obtained. 
Table 3. Effect of C-Section on Early Neonatal Mortality

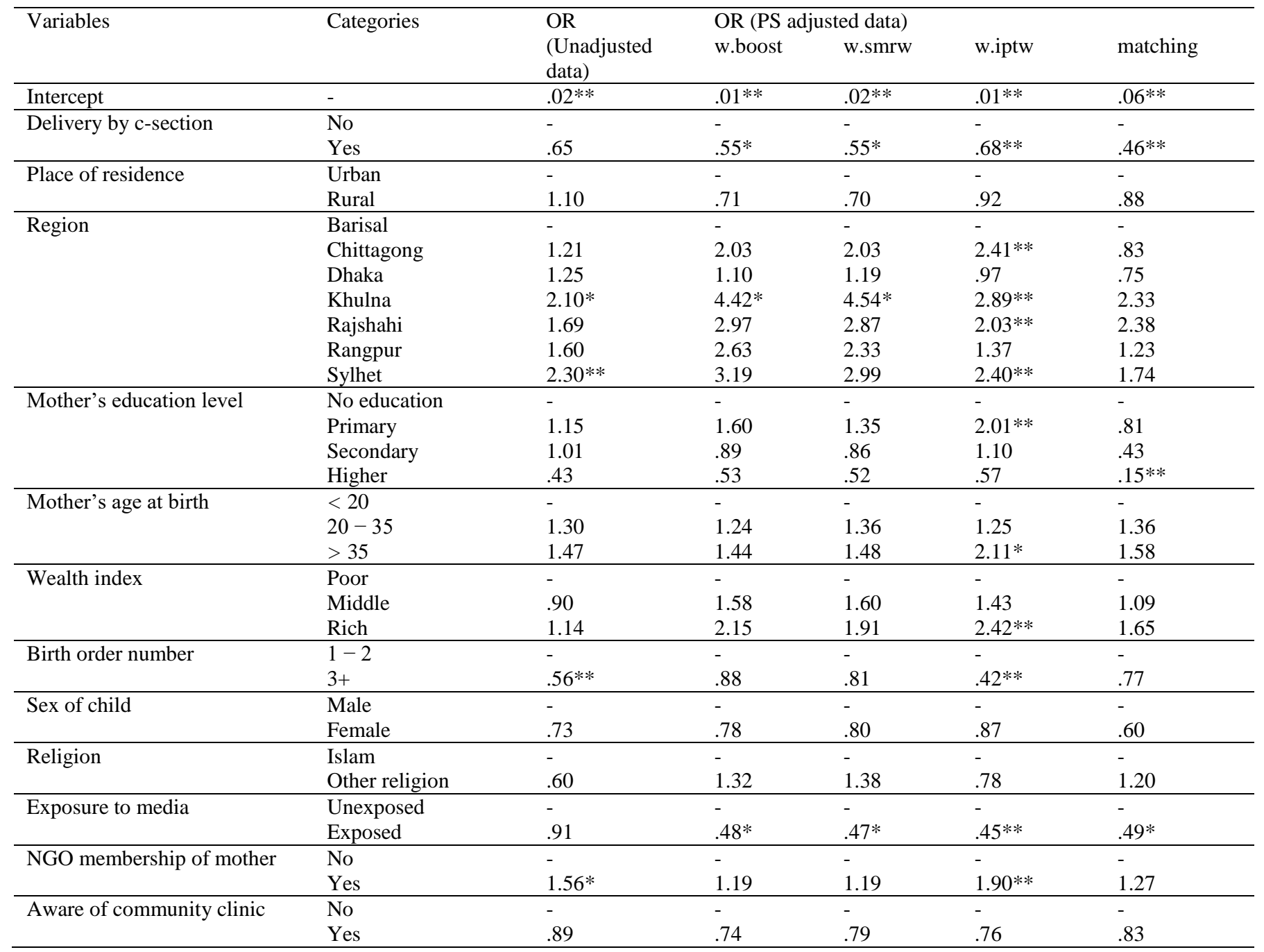

p-value $<0.05(* *), \mathrm{p}$-value $<0.10(*)$

\section{Effect of c-section on neonatal mortality}

Next, Table 4 compares the Odds Ratio (OR) of neonatal mortality obtained from standard logistic model with various propensity score adjusted methods and corresponding statistical significance.
After the adjustment by w.boost, it is also found insignificant association between c-section and neonatal mortality. The result shows that a non-c-section child is 1.61 times more likely to have neonatal mortality compared to a c-section child, keeping all other covariates at a fixed level.

Table 4. Effect of C-Section on Neonatal Mortality

\begin{tabular}{|c|c|c|c|c|c|c|}
\hline \multirow[t]{2}{*}{ Variables } & \multirow[t]{2}{*}{ Categories } & \multirow{2}{*}{$\begin{array}{l}\text { OR } \\
\text { (Unadjusted data) }\end{array}$} & \multicolumn{3}{|c|}{ OR (PS adjusted data) } & \multirow[b]{2}{*}{ matching } \\
\hline & & & w.boost & w.smrw & w.boost & \\
\hline Intercept & - & $.02 * *$ & $.01 * *$ & $.01 * *$ & $.01 * *$ & $.05 * *$ \\
\hline \multirow[t]{2}{*}{ Delivery by c-section } & No & - & - & - & - & - \\
\hline & Yes & .70 & .62 & $.61 *$ & $.76 * *$ & $.48 * *$ \\
\hline \multirow[t]{2}{*}{ Place of residence } & Urban & - & - & - & - & - \\
\hline & Rural & 1.01 & .73 & .70 & .98 & .80 \\
\hline \multirow[t]{7}{*}{ Region } & Barisal & - & - & - & - & - \\
\hline & Chittagong & 1.63 & 2.98 & 3.02 & $3.20 * *$ & 1.59 \\
\hline & Dhaka & 1.49 & 1.39 & 1.54 & 1.24 & 1.05 \\
\hline & Khulna & $2.44 * *$ & $4.63 *$ & $4.94 * *$ & $3.16^{* *}$ & 2.79 \\
\hline & Rajshahi & $2.23 *$ & 3.12 & 3.06 & $2.34 * *$ & $3.08 *$ \\
\hline & Rangpur & 1.89 & 2.79 & 2.54 & 1.54 & 1.64 \\
\hline & Sylhet & $2.99 * *$ & $4.46 * *$ & $4.20 *$ & $4.42 * *$ & 2.14 \\
\hline
\end{tabular}




\begin{tabular}{|c|c|c|c|c|c|c|}
\hline \multirow[t]{2}{*}{ Variables } & \multirow[t]{2}{*}{ Categories } & \multirow{2}{*}{$\begin{array}{l}\text { OR } \\
\text { (Unadjusted data) }\end{array}$} & \multicolumn{2}{|c|}{ OR (PS adjusted data) } & \multirow[b]{2}{*}{ w.boost } & \multirow[b]{2}{*}{ matching } \\
\hline & & & w.boost & w.smrw & & \\
\hline \multirow[t]{4}{*}{ Mother's education level } & No education & - & - & - & - & - \\
\hline & Primary & 1.05 & 1.77 & 1.69 & $2.14 * * 1.08$ & 1.29 \\
\hline & Secondary & .84 & .95 & .93 & .65 & .51 \\
\hline & Higher & $.37 *$ & .58 & .57 & & $.21 * *$ \\
\hline \multirow[t]{3}{*}{ Mother's age at birth } & $<20$ & - & - & - & - & - \\
\hline & $20-35$ & 1.38 & 1.25 & 1.41 & $1.43 * *$ & 1.38 \\
\hline & $>35$ & 1.42 & 1.31 & 1.37 & $2.09 *$ & 1.37 \\
\hline \multirow[t]{3}{*}{ Wealth index } & Poor & - & - & - & - & - \\
\hline & Middle & .91 & 1.42 & 1.48 & 1.23 & 1.25 \\
\hline & Rich & 1.09 & 1.71 & 1.62 & $1.99 * *$ & 1.42 \\
\hline \multirow[t]{2}{*}{ Birth order number } & $1-2$ & - & - & - & - & - \\
\hline & $3+$ & $.56 * *$ & .86 & .78 & $.36 * *$ & .74 \\
\hline \multirow[t]{2}{*}{ Sex of child } & Male & - & - & - & - & - \\
\hline & Female & $.70 *$ & .88 & .88 & 1.01 & .65 \\
\hline \multirow[t]{2}{*}{ Religion } & Islam & - & - & - & - & - \\
\hline & Other religion & .65 & 1.14 & 1.19 & .66 & .93 \\
\hline \multirow[t]{2}{*}{ Exposure to media } & Unexposed & - & - & - & - & - \\
\hline & Exposed & .78 & $.51 *$ & $.48^{*}$ & $.45 * *$ & $.48 *$ \\
\hline \multirow[t]{2}{*}{ NGO membership of mother } & No & - & - & - & - & - \\
\hline & Yes & 1.31 & 1.15 & 1.13 & $2.20 * *$ & 1.13 \\
\hline \multirow[t]{2}{*}{ Aware of community clinic } & No & - & - & - & - & - \\
\hline & Yes & .78 & .71 & .77 & $.66 * *$ & .87 \\
\hline
\end{tabular}

p-value $<0.05(* *)$, p-value $<0.10(*)$

Table 5. Effect of C-Section on Early Initiation of Breastfeeding

\begin{tabular}{|c|c|c|c|c|c|c|}
\hline \multirow[t]{2}{*}{ Variables } & \multirow[t]{2}{*}{ Categories } & \multirow{2}{*}{$\begin{array}{l}\text { OR } \\
\text { (Unadjusted data) }\end{array}$} & \multicolumn{2}{|c|}{ OR (PS adjusted data) } & \multirow[b]{2}{*}{ w.boost } & \multirow[b]{2}{*}{ matching } \\
\hline & & & w.boost & w.smrw & & \\
\hline Intercept & - & $1.30 *$ & 1.27 & 1.20 & 1.20 & 1.11 \\
\hline \multirow[t]{2}{*}{ Delivery by c-section } & No & - & - & - & - & - \\
\hline & Yes & $.32 * *$ & $.32 * *$ & $.32 * *$ & $.33 * *$ & $.32 * *$ \\
\hline \multirow{2}{*}{ Place of residence } & Urban & - & - & - & - & - \\
\hline & Rural & 1.04 & 1.11 & 1.11 & 1.01 & 1.03 \\
\hline \multirow[t]{7}{*}{ Region } & Barisal & - & - & - & - & - \\
\hline & Chittagong & $.72 * *$ & .78 & .76 & 1.08 & .95 \\
\hline & Dhaka & .95 & 1.07 & 1.07 & 1.04 & 1.14 \\
\hline & Khulna & $.79 *$ & .75 & .76 & $.85^{*}$ & .88 \\
\hline & Rajshahi & 1.01 & .91 & .95 & 1.07 & 1.01 \\
\hline & Rangpur & 1.23 & 1.07 & 1.14 & $1.34 * *$ & 1.37 \\
\hline & Sylhet & $1.28 *$ & $1.57 * *$ & $1.61^{* *}$ & $1.64 * *$ & $1.65 * *$ \\
\hline \multirow[t]{4}{*}{ Mother's education level } & No education & - & - & - & - & - \\
\hline & Primary & .91 & .96 & .99 & 1.03 & .97 \\
\hline & Secondary & .90 & .90 & .92 & .96 & .89 \\
\hline & Higher & .79 & .78 & .78 & $.83 *$ & .77 \\
\hline \multirow{3}{*}{ Mother's age at birth } & $<20$ & - & - & - & - & - \\
\hline & $20-35$ & .99 & 1.06 & 1.04 & .98 & 1.08 \\
\hline & $>35$ & 1.02 & .84 & .71 & .97 & 1.06 \\
\hline \multirow[t]{3}{*}{ Wealth index } & Poor & - & - & - & - & - \\
\hline & Middle & 1.10 & 1.20 & 1.24 & 1.34 & 1.20 \\
\hline & Rich & 1.15 & 1.21 & 1.23 & $1.27 * *$ & 1.19 \\
\hline \multirow[t]{2}{*}{ Birth order number } & $1-2$ & - & - & - & - & - \\
\hline & $3+$ & .90 & .87 & .89 & $.82 * *$ & .95 \\
\hline \multirow[t]{2}{*}{ Sex of child } & Male & - & - & - & - & - \\
\hline & Female & $.1 .10^{*}$ & 1.05 & 1.06 & $1.00 * *$ & 1.04 \\
\hline \multirow[t]{2}{*}{ Religion } & Islam & - & - & - & - & - \\
\hline & Other religion & .89 & .85 & .83 & .98 & .89 \\
\hline \multirow[t]{2}{*}{ Exposure to media } & Unexposed & - & - & - & - & - \\
\hline & Exposed & 1.08 & .96 & .98 & $.86 * *$ & 1.07 \\
\hline \multirow[t]{2}{*}{ NGO membership of mother } & No & - & - & - & - & - \\
\hline & Yes & 1.03 & .99 & .99 & 1.00 & .92 \\
\hline \multirow[t]{2}{*}{ Aware of community clinic } & No & - & - & - & - & - \\
\hline & Yes & $1.17 * *$ & $1.23 * *$ & $1.24 * *$ & $1.27 * *$ & $1.21 *$ \\
\hline
\end{tabular}

p-value $<0.05(* *)$, p-value $<0.10(*)$ 
w.smrw, w.iptw and matching methods reveals that there is significant association between c-section and neonatal mortality at $10 \%, 5 \%$ and $5 \%$ level of significance respectively. w.smrw, w.iptw and matching methods shows that a c-section child has respectively 39\%, 24\% and 52\% lower odds of having the Neonatal mortality than a non-csection child, keeping all other covariates at a fixed level.

Majority of the adjusted methods support the significant association between c-section delivery and neonatal mortality. It can be concluded that there is a significant association between the variables and the result has improved because of removing the effect of confounding by different propensity adjusted methods (e.g. w.smrw, w.iptw, matching) and applying them to the model.

\section{Effect of c-section on early initiation of breastfeeding}

Table 5 shows the comparison of the Odds Ratio (OR) of early initiation of breastfeeding obtained from standard logistic regression model and propensity score adjusted methods, and corresponding statistical significance.

However, when bivariate analysis using propensity score adjusted methods were performed the results show that the effect of c-section on outcome variables are almost like the multivariate propensity score adjusted methods. For the convenience of comparison with the standard multivariate logistic model, only the result of multivariate propensity adjusted methods were shown.

\section{Conclusion}

After applying propensity score methods, it is observed that c-section has significant effect on early neonatal mortality in all adjusted methods. C-section has also significant effect on neonatal mortality in all adjusted methods except w.boost. It was also found that $\mathrm{C}$-section has significant effect on early initiation of breastfeeding in both standard logistic and propensity score adjusted methods.

In all cases, it is found that Children who are delivered by c-section have lower odds of early neonatal mortality, neonatal mortality, and early initiation of breastfeeding compared to the children who are not delivered by csection.

If balance with respect to other pretreatment variables is ignored, it is observed that c-section has no significant impact on early neonatal mortality, neonatal mortality, but has significant association with early initiation of breastfeeding. However, when balance is achieved using propensity score, c-section is significantly associated with early neonatal mortality, neonatal mortality, and early initiation of breastfeeding.

For further study, two other methods, stratification and covariate adjustment, can be used to estimate the true effect of treatment. Furthermore, only 11 pretreatment variables were included in the dataset. More pretreatment variables may be included for greater reduction in confounding effect.
A detailed simulation study can be conducted with varying the degree of the effect of confounders and examine which method can better reduce the effect of confounders.

\section{References}

1. Bangladesh Demographic and Health Survey (BDHS), 2014. NIPORT, Dhaka, Bangladesh; Mitra and Associates, Dhaka, Bangladesh.

2. World Health Organization, 2009. Monitoring Emergency Obstetric Care: A Handbook. Geneva: World Health Organization.

3. Rowe-Murray H.J., and J.R.W. Fisher, 2002. Baby friendly hospital practices: cesarean section is a persistent barrier to early initiation of breastfeeding. Birth (Berkeley, Calif), 29(2), 124-31.

4. MacDorman, M.F., E. Declercq, F. Menacker, and M.H. Malloy, 2006. Infant and neonatal mortality for primary caesarean and vaginal births to women with "no indicated risk", United States, 1998-2001 Birth Cohorts. Birth, 33, 175-82.

5. Rosenbaum, P.R., and D.B. Rubin, 1983. The central role of the propensity score in observational studies for causal effects. Biometrika, 70, 41-55.

6. Lee, B.K., J. Lessler, and E.A. Stuart, 2010. Improving propensity score weighting using machine learning. Statistics in Medicine, 29, 337-346.

7. McCaffrey, D.F., G. Ridgeway, and A.R. Morral, 2004. Propensity score estimation with boosted regression for evaluating causal effects in observational studies. Psychological Methods, 9, 403-425.

8. Setoguchi, S., S. Schneeweiss, M.A. Brookhart, R.J. Glynn, and E.F. Cook, 2008. Evaluating uses of data mining techniques in propensity score estimation: A simulation study. Pharmacoepidemiology and Drug Safety, 17, 546-555.

9. Hong, J., and B. Yu, 2008. Effects of kindergarten retention on children's social emotional development: An application of propensity score method to multivariate, multilevel data. Developmental Psychology, 44, 407-421.

10. Ye, Y., and L.A. Kaskutas, 2009. Using propensity scores to adjust for selection bias when assessing the effectiveness of Alcoholics Anonymous in observational studies. Drug and Alcohol Dependence, 104, 56-64.

11. Wyse, A. E., V. Keesler, and B. Schneider, 2008. Assessing the effects of small school size on mathematics achievement: A propensity score-matching approach. Teachers College Record, 110, 1879-1900.

12. Staff, J., M.E. Patrick, E. Loken, and J.L. Maggs, 2008. Teenage alcohol use and educational attainment. Journal of Studies on Alcohol and Drugs, 69, 848-858.

13. Zaoutis T. E., J. Argon, J. Chu, J. A. Berlin, T.J. Walsh, and C. Feudtner, 2005. The epidemiology and attributable outcomes of candidemia in adults and children hospitalized in the United States: a propensity analysis. Clinical Infectious Diseases: An official publication of the Infectious Diseases Society of America. Nov 1;41(9),1232-9.

14. Stuart, E.A., and K.M. Green, 2008. Using full matching to estimate causal effects in nonexperimental studies: Examining the relationship between adolescent marijuana use and adult outcomes. Developmental Psychology, 44(2), 395-406. 
